\title{
Unlocking Schizophrenia with Imaging Markers Related to Striatal Dysfunction
}

A round four in a thousand people worldwide suffer from schizophrenia. according to scientific estimates. The disease affects people from all walks of life, including Vincent van Gogh, the painter Agnes Martin, mathematician John Nash and Eduard Einstein, a son of the great physicist. The disease touches men and women equally.

Despite its prevalence, however. schizophrenia has remained a mystery. Diagnosis has relied on patient behavior, such as hallucinations, delusions and disordered thinking. rather than on quantitative biomarkers. As a result, psychiatrists' ability to objectively diagnose and treat people with the illness has been impaired.

New research, led by Prof. LIU Bing and Prof. JIANG Tianzi from the Institute of Automation of the Chinese Academy of Sciences (CASIA) and their collahorators, may change this situation, though. The scientists have recently developed a novel imaging marker that may help in the personalized medicine of psychiatric disorders.

The study, published in Nature Medicine on Mar. 2:3. shows that abnormal striatal function can be a promising biomarker for the diagnosis of schizophrenia and treatment response.

In their search for suitable biomarkers, the scientists collected multimodal neuroimaging data from a total of 1.100 individuals with schizophrenia and healthy controls from 2010 to 15 .

Using the large dataset, the researchers first proposed the concept of "functional striatal abnormalities." They then used artificial intelligence technology on resting state MIRI data to map striatal dysfunction at the individual level.

The researchers showed, for the first time, that striatal dysfunction was effective in distinguishing schizophrenia patients and that such dysfunction was also responsible for poorer antipsychotic response.

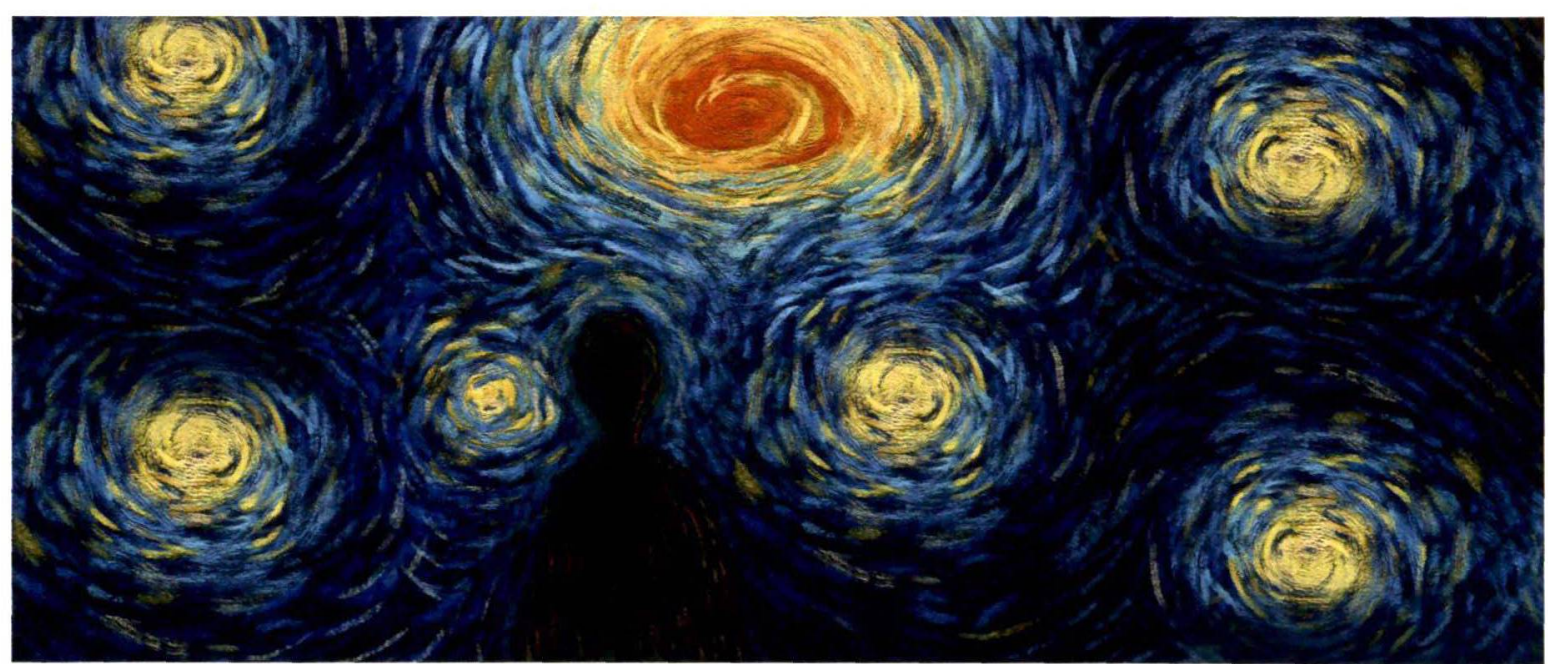

Newly discovered imaging markers related to the abnormalities of striatum may illuminate the stealth of schizophrenia. (Image by WANG Guoyan \& CHEN Lei from the University of Science and Technology of China) 


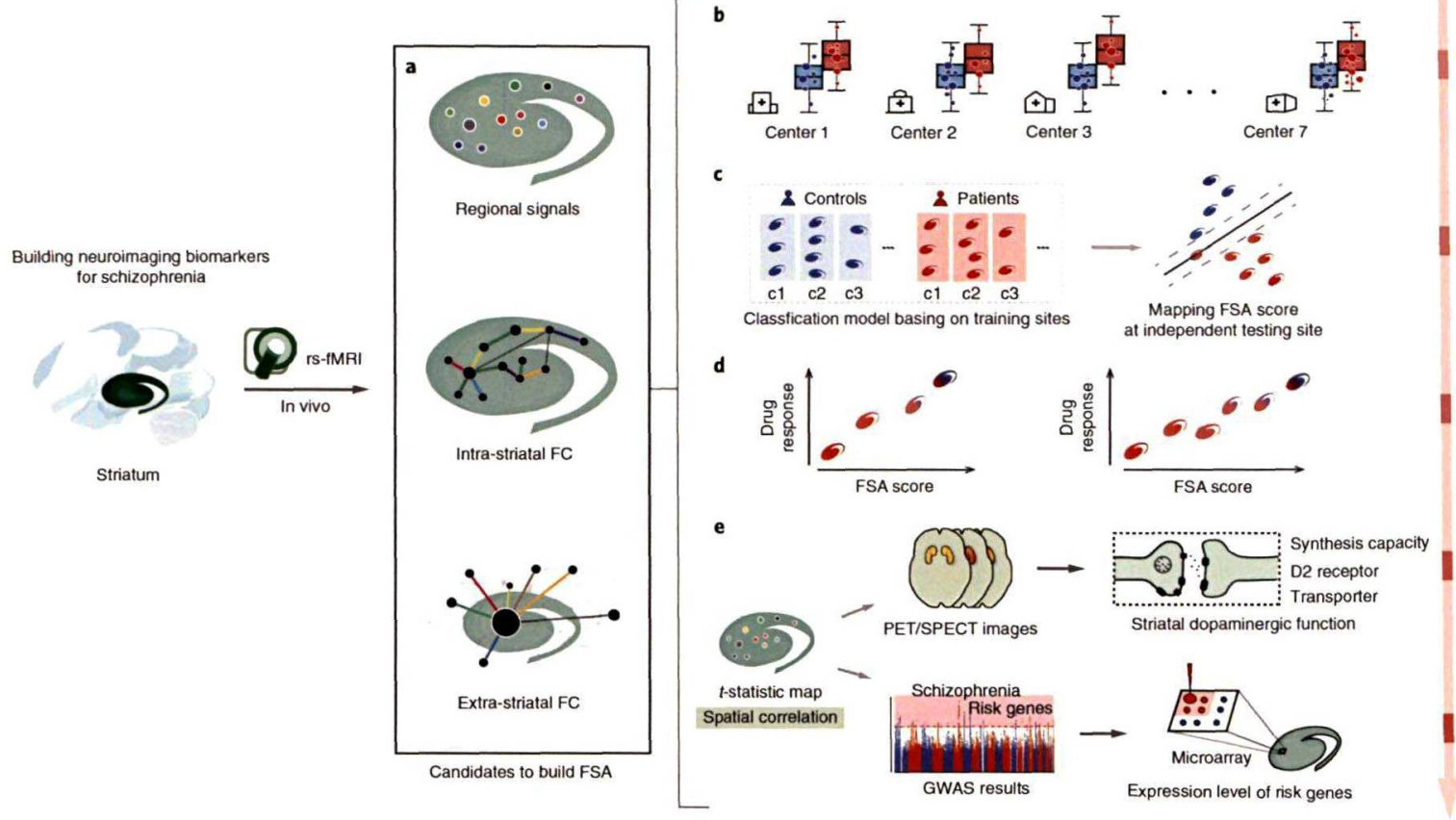

Schema of development, testing and validation of the biomarker related to functional striatal abnormalities. (Credit: Noture Medicine)

Based on the newly developed biomarker, the researchers extended their research to other neuropsychiatric disorders. They showed that individuals with bipolar disorder also showed striatal dysfunction that overlapped with the dysfunction associated with schizophrenia.

After combining different levels of data. the researchers suggested that striatal dysfunction is related to the dopaminergic system and polygenic genetic risk for schizophrenia.

The study also evaluated using this biomarker to predict diagnostic labels and treatment responses across several different hospitals.

The scientists hope research on the biological underpinnings of psychiatric disorders will increase understanding of disease mechanisms as well as guide new drug development.

Website of the research tool for calculating functional striatal abnormalities score is as follows: https://www. szbiomarkers.ned

The research was supported by the National Basic Research Program of China, the National Key Research and Development Program. the Leading Projects Program of the Chinese Academy of Sciences and the Natural Science Foundation of China.

\section{Contact}

ZHANG Xiaohan, PIO, Institute of Automation, Chinese Academy of Sciences

Email: xiaohan.zhang@ia.ac.cn

\section{Reference}

Ang Li, Andrew Zalesky, Weihua Yue, Oliver Howes, Hao Yan, Yong Liu, Lingzhong Fan, Kirstie J. Whitaker, Kaibin Xu, Guangxiang Rao, Jin Li, Shu Liu, Meng Wang, Yuaing Sun, Ming Song, Peng Li, Jun Chen, Yunchun Chen, Huaning Wang, Wenming Liu, Zhigang Li, Yongfeng Yang, Hua Guo, Ping Wan, Luxian Lv, Lin Lu, Jun Yan, Yuqing Song, Huiling Wang, Hongxing Zhang, Huawang Wu, Yuping Ning, Yuhui Du, Yuqi Cheng, Jian $\mathrm{Xu}$, Xiufeng Xu, Dai Zhang, Xiaoqun Wang, Tianzi Jiang*, Bing Liu*, (2020) A neuroimaging biomarker for striatal dysfunction in schizophrenia. Nature Medicine. doi: 10.1038/s41591-020-0793-8. 